\title{
A Case of a Cellular Neurothekeoma Presenting with Headaches and Review of the Literature
}

\author{
Benjamin Bashline $^{a} \quad$ Megan Morrison ${ }^{b}$ Hyuk Chol Cha ${ }^{b} \quad$ James Ramirez ${ }^{c}$ \\ ${ }^{a}$ Firelands Regional Medical Center, Sandusky, Ohio, and Departments of ${ }^{b}$ Dermatology \\ and ' Dermatopathology, St. Joseph Mercy Hospital, Ann Arbor, Mich., USA
}

\author{
Key Words \\ Cellular neurothekeoma $\cdot$ Scalp tumor $\cdot$ Headache
}

\begin{abstract}
A 12-year-old female presented with a 1-year history of a slow-growing lesion on the frontal scalp. The patient reported intermittent headaches increasing in frequency localized under the lesion. Physical exam revealed a firm, fixed, 5-mm orange-pink papule on the right frontal scalp. Excisional biopsy was performed using a 6-mm punch biopsy, after which the patient reported a cessation of headaches. Histopathology showed a diffuse proliferation of spindled and epithelioid cells within the superficial and deep reticular dermis demonstrating a nested as well as fascicular growth pattern. There was also focal myxoid dermal change. Immunohistochemical staining showed the tumor to be strongly positive for NK1C3 and negative for S-100. A diagnosis of cellular neurothekeoma was made. Cellular neurothekeomas are slowgrowing, asymptomatic nodules that most frequently appear on the head and neck of young adults. Cellular neurothekeomas rarely present with symptoms beyond mild tenderness upon palpation. Our case represents a unique presentation of a neurothekeoma of the scalp with complaint of headaches, previously unpublished in the literature.

(c) 2014 S. Karger AG, Basel
\end{abstract}

\section{Introduction}

Neurothekeomas are uncommon benign skin neoplasms of uncertain histogenesis. Cellular neurothekeomas are considered a variant of neurothekeomas, with distinct microscopic and immunohistochemical features. Lesions typically appear on the head and upper extremities of young adults. Here we report a case of a cellular neurothekeoma on the scalp of a 12-year-old girl presenting with intermittent headaches. 
Fig. 1. A 5-mm orange-pink papule on the right frontal scalp.
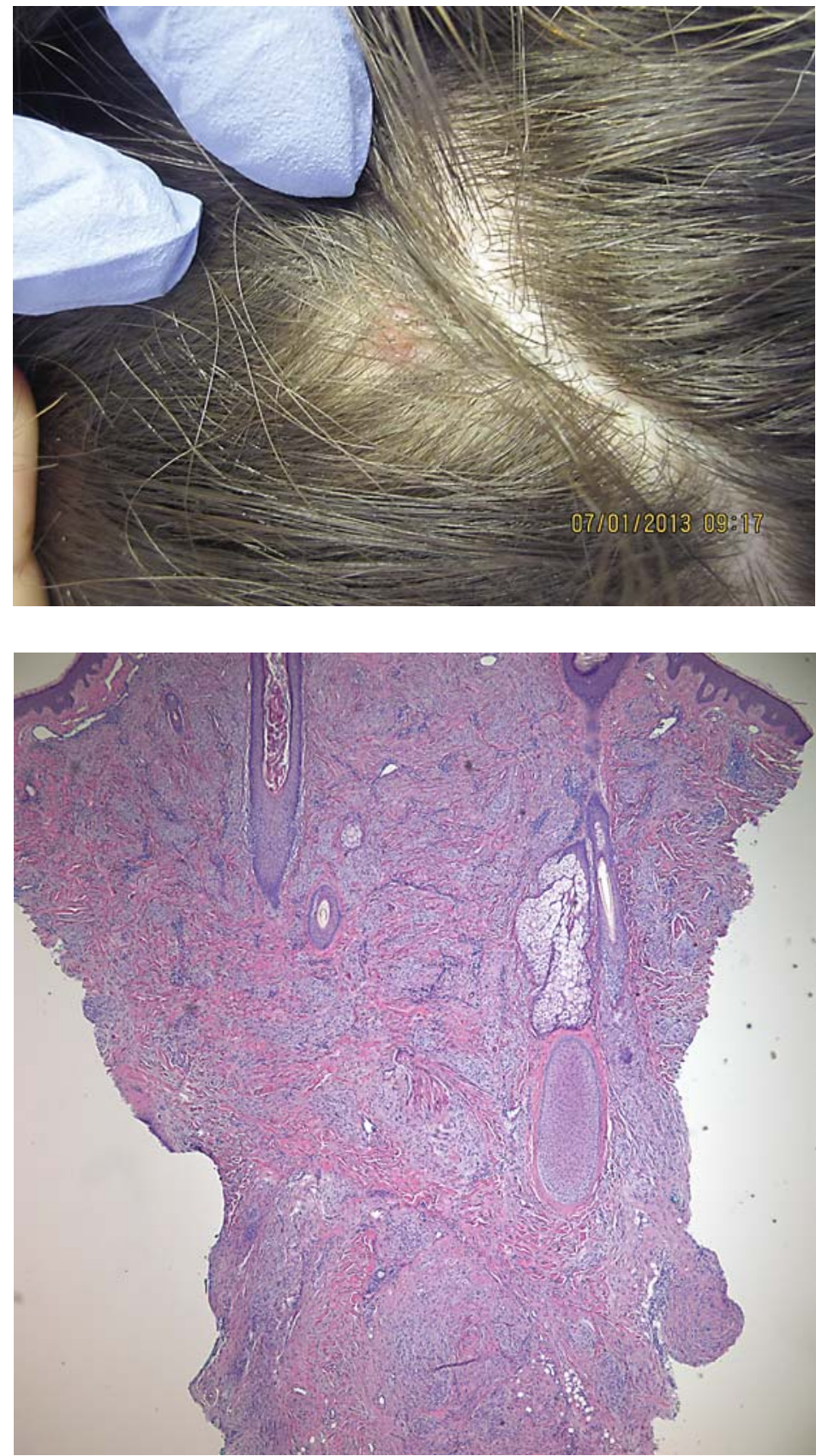

Fig. 2. Low power reveals numerous nests and fascicles of cells intersecting the collagen bundles of the superficial and deep reticular dermis. HE. $\times 4$. 
Fig. 3. The nests and fascicles are composed of large, oval to spindled cells with abundant amphophilic cytoplasm and little nuclear cytologic pleomorphism; occasional cells possess smudgy hyperchromatic nuclei. HE. $\times 10$.

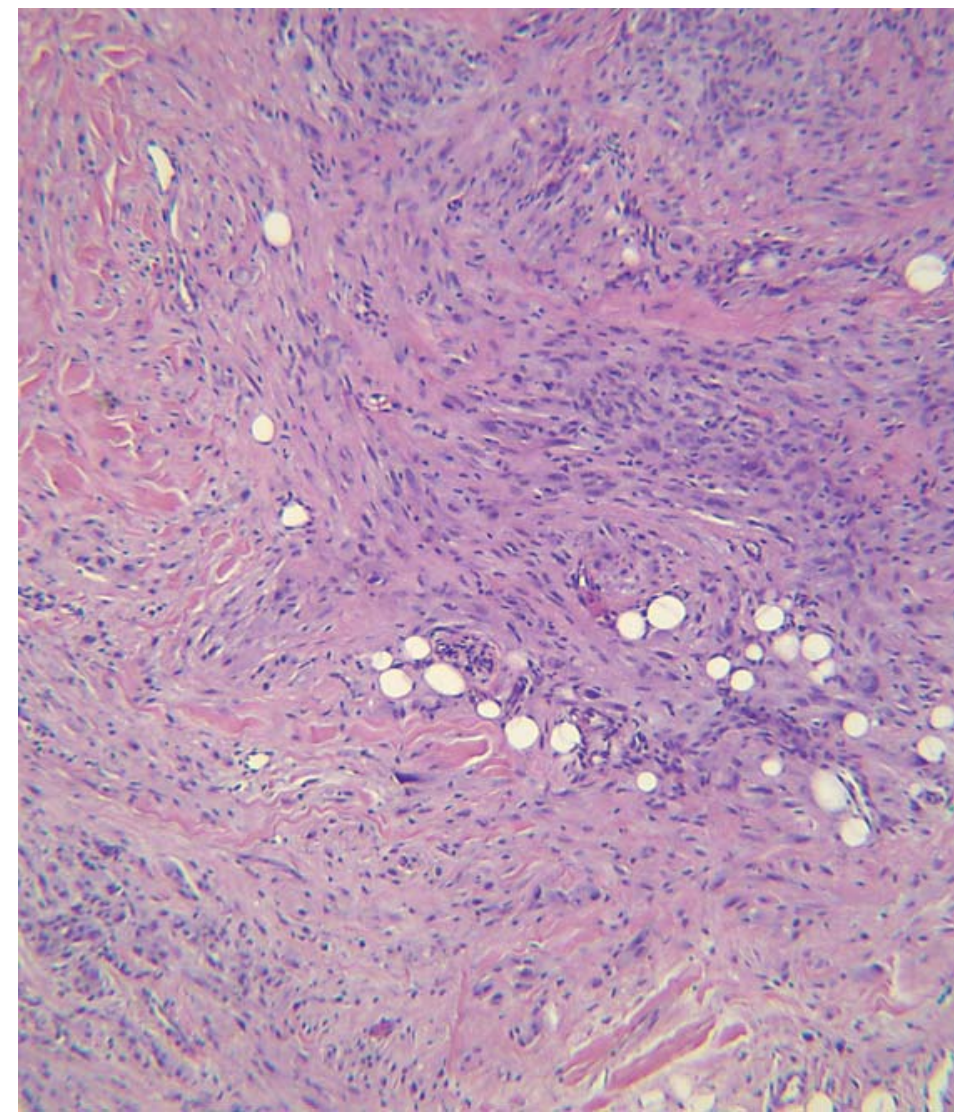

(fig. 2-4). Most cells were large cells and contained amphophilic cytoplasm and bland nuclei. Occasional cells contained nuclei that demonstrated smudgy hyperchromasia. There were also focal myxoid stromal changes. Immunohistochemical staining showed the tumor to be strongly positive for NK1C3 (fig. 5) and negative for S-100. These findings confirmed a diagnosis of cellular neurothekeoma. Conservative re-excision with 2-mm margins was performed, and the patient reported a cessation of headaches after removal of the lesion.

\section{Discussion}

Neurothekeoma was first described by Harkin and Reed [1] in 1969 as a 'rare neoplasm arising in endoneurium of peripheral nerves and characterized by an abundant mucoid matrix' and called 'myxoma of the nerve sheath'. The term neurothekeoma was first used in 1980 by Gallager and Helwig [2]. Neurothekeomas were initially divided into three histological variants: classical (myxoid), cellular, and mixed, with mixed type tumors showing microscopic features of both classical and cellular variants [3]. The classical lesions are characterized by myxoid stroma containing well-circumscribed nests of epithelioid and spindled cells. These subtypes generally stain positive for S-100, collagen type IV, and nerve growth factor, and do not stain for epithelial membrane antigen or markers of histiocytic differentiation [4]. These lesions are best considered nerve sheath myxomas, as they demonstrate features of peripheral nerve differentiation including consistent S-100 positivity. 
Fig. 4. The nests and fascicles are composed of large, oval to spindled cells with abundant amphophilic cytoplasm and little nuclear cytologic pleomorphism; occasional cells possess smudgy hyperchromatic nuclei. HE. $\times 20$.

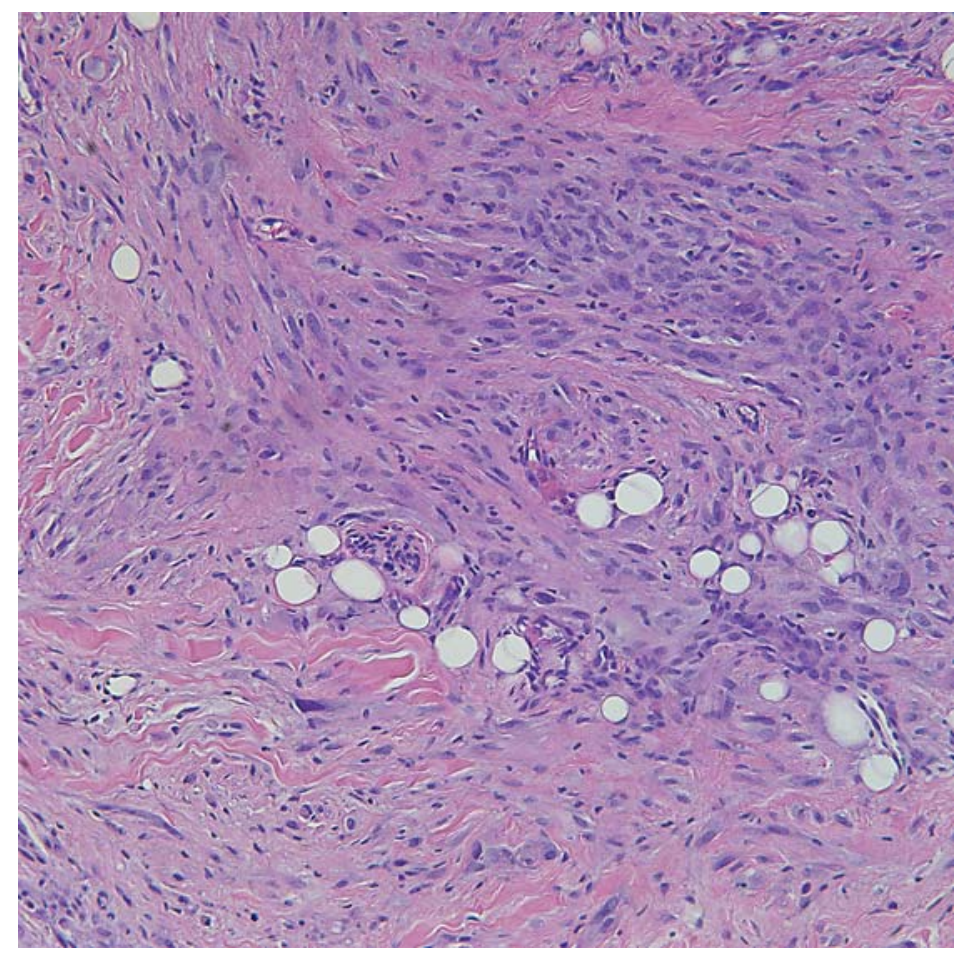

Fig. 5. Immunohistochemical staining positive for antibodies to NK1C3 $(\times 20)$.

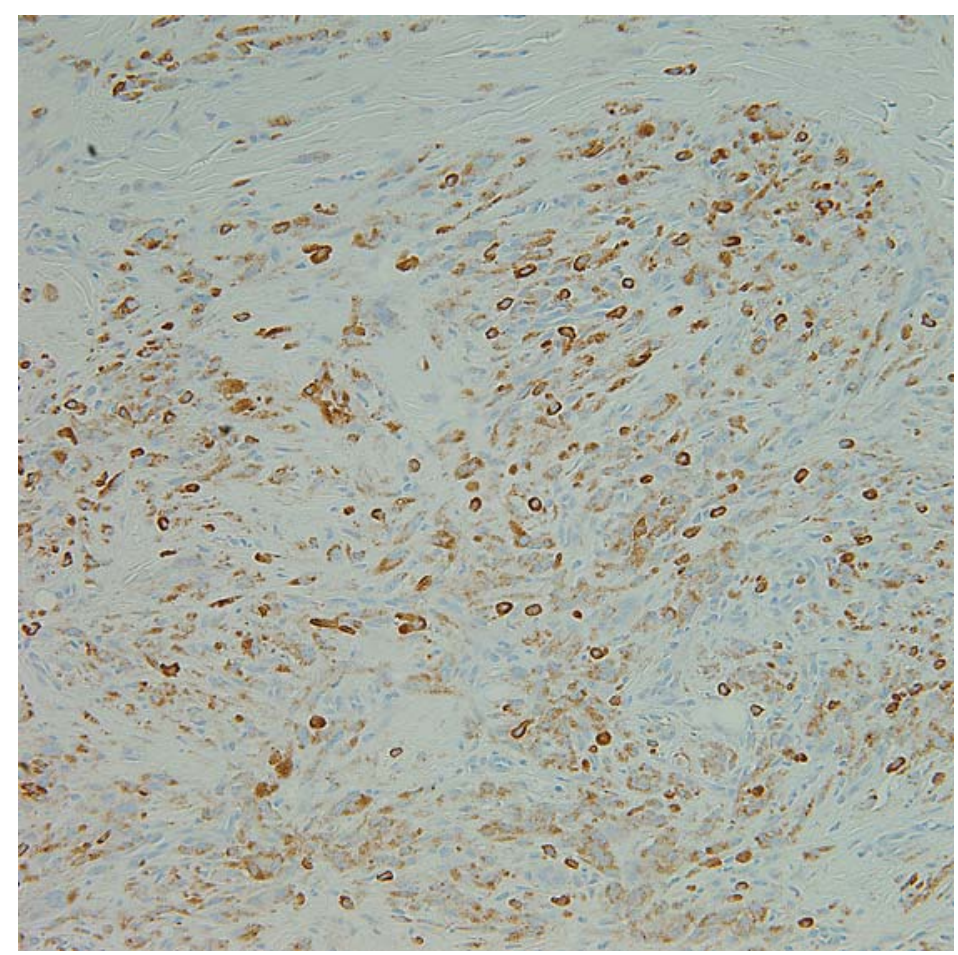

Cellular neurothekeomas were first described by Rosati in 1986, but the histogenesis of the neoplasm remains ill-defined [5]. They lack distinctive microscopic and immunohistochemical features consistent with neural differentiation, and contain a mixture of cell lines with immature features of fibroblasts, Schwann cells, myofibroblasts, perineural cells, smooth muscle cells, and histiocytes [6]. The tumors consist of poorly circumscribed nests 
and fascicles of epithelioid cells with pale eosinophilic cytoplasm. The cells stain positively for NK1C3 and Ki-M1p, and do not stain for S-100, collagen type IV, and nerve growth factor [7]. Zelger et al. [8] have suggested that cellular neurothekeomas may be a variant of dermatofibroma due to many similar features, including benign course with rare recurrence after excision, lack of visceral involvement, tendency to occur in young females, variable immunochemistry, predominately dermal locations and peripherally accentuated sclerotic collagen.

The variability of microscopic and immunohistochemical findings in cellular neurothekeoma has created problems in establishing a universal set of criteria for diagnosis, leaving a broad differential diagnosis in many cases. Argenyi et al. [9] suggested that reevaluation of these neoplasms is necessary, as well as a return to the original moniker of neural sheath myxoma for classic lesions, and the development of a new term to describe the cellular variants. In a study conducted by Hornick and Fletcher [10], 133 cases of cellular neurothekeomas were reviewed from 1987 to 2003. Of the cases reviewed, the mean age of tumor onset was 25 years, and $84 \%$ of the patients were under the age of 40 , with a 1:1.8 male-to-female ratio. Thirty-five percent of lesions were found on the upper extremities, and $33 \%$ on the head or neck, with a mean size of $1.1 \mathrm{~cm}$. All tumors were found to be reactive for NK1C3 and nonreactive for S-100 immunostaining [10]. The significance of atypia (high mitotic rate, pleomorphism, and infiltration of the subcutaneous tissues) in these tumors has yet to be determined, although studies have suggested minimal clinical impact [10].

Our case demonstrates typical microscopic and immunohistochemical features associated with cellular neurothekeomas. The specimen stained positively for NK1C3 and did not stain for S-100, with histology showing spindled and epithelioid cells in nests and fascicles, all consistent with the diagnosis of cellular neurothekeoma. The complaint of headaches by our patient is a unique aspect of our case, as these lesions are most commonly associated with mild tenderness of the lesion upon palpation, and only cases featuring intracranial neurothekeomas have been associated with significant headaches [11].

The connection between extracranial tumors and headaches has yet to be clearly defined, although intracranial processes are commonly associated with headaches secondary to mass effect. Several cases of extracranial tumors producing headaches have been published in the literature. Murali et al. [12] report a case of severe headaches caused by sebaceous cell carcinoma of the scalp, which resolved after removal of the lesion. Kawale et al. [13] detail a case involving headaches secondary to a lipoma of the scalp compressing a branch of the greater occipital nerve in a patient with adiposis dolorosa. We believe the headaches in our case may have been associated with the compression of a small cutaneous nerve, which was alleviated upon removal of the lesion.

In summary, cellular neurothekeomas are uncommon, benign tumors of controversial origin, typically found on the head, neck or upper extremities of young adults. Our case represents a unique presentation of an extracranial neurothekeoma of the scalp with complaint of headaches, previously unpublished in the literature.

\section{References}

1 Harkin JC, Reed RJ: Solitary benign nerve sheath tumors; in Harkin JC, Reed RJ (eds): Tumors of the Peripheral Nervous System. Atlas of Tumor Pathology. Washington, Armed Forces Institute of Pathology, 1969, pp 60-64.

$>2$ Gallager RL, Helwig EB: Neurothekeoma - a benign cutaneous tumor of neural origin. Am J Clin Pathol 1980; 74:759-764.

-3 Müller CSL, Tilgen W, Kutzner H, Pföhler C: Recurring mixed-type neurothekeoma of the face. Dermatoendocrinol 2009;1:220-222.

-4 Papadopoulos EJ, Cohen PR, Hebert AA: Neurothekeoma: report of a case in an infant and review of the literature. J Am Acad Dermatol 2004;50:129-134. 
Bashline et al.: A Case of a Cellular Neurothekeoma Presenting with Headaches and Review of the Literature

5 Ward JL, Prieto VG, Joseph A, Chevray P, Kronowitz S, Sturgis EM: Neurothekeoma. Otolaryngol Head Neck Surg 2005;132:86-89.

6 Pan HY, Tseng SH, Weng CC, Chen Y: Cellular neurothekeoma of the upper lip in an infant. Pediatr Neonatol, Epub ahead of print.

7 Rudolph P, Schubert C: Myxoid cellular neurothekeoma. Am J Dermatopathol 2002;24:92-93.

-8 Zelger ZB, Steiner H, Kutzner H, Maier H, Zelger B: Cellular 'neurothekeoma': an epithelioid variant of dermatofibroma? Histopathology 1998;32:414-422.

-9 Argenyi ZB, LeBoit PE, Santa Cruz DJ, Swanson PE, Kutzner H: Nerve sheath myxoma (neurothekeoma) of the skin: light microscopic and immunohistochemical reappraisal of the cellular variant. J Cutan Pathol 1993;20: 294-303.

10 Hornick JL, Fletcher CD: Cellular neurothekeoma: detailed characterization in a series of 133 cases. Am J Surg Pathol Mar 2007;31:329-340.

11 Alexandru D, Satvadey R, So W: Neurothekeoma in the posterior fossa: case report and literature review. Perm J 2012;16:63-64.

12 Murali V, Bhavarajua K, Shamim SE, Naik VR, Shaari S: Sebaceous cell carcinoma of the scalp - a rare presentation. Malays J Med Sci 2007;14:67-70.

13 Kawale J, Mahore A, Dange N, Bhoyar K: Adiposis delorosa of scalp presenting with severe headache: an unusual case. J Headache Pain 2010;11:539-541. 